\title{
Epidémies de Choléra en Afrique Sub-Saharienne: Revue documentaire de 2010 à 2016
}

\author{
Alkassoum Salifou Ibrahim (MD, Msc, PhD candidat) \\ Département de Santé Publique, Faculté des Sciences de la Santé, \\ Université Abdou Moumouni, Niamey, Niger \\ Doutchi Mahamadou (MD, MPH Infectious Disease)
}

Faculté des Sciences de la Santé, Université de Zinder, Niger

Amadou Harouna (MD, Infectious Disease)

Département de Santé Publique, Faculté des Sciences de la Santé,

Université Dan Dicko Dan Koulodo, Maradi, Niger

Brah Souleymane (MD, MI)

Département de médecine et spécialités médicales, Faculté des Sciences de

la Santé, Université Abdou Moumouni, Niamey, Niger

Djibo Issifou (MD, Msc, PhD candidat)

Direction de la Surveillance et la Riposte aux Epidémies, MSP,

Niamey, Niger

Ibrahim Mahaman Lamine (Vet. PhD)

Lagaré A. (Biol, PhD candidat)

Centre de Recherche Médicale et Sanitaire de Niamey, Niger

Adehossi Eric (MD, MI)

Département de médecine et spécialités médicales, Faculté des Sciences de

la Santé, Université Abdou Moumouni, Niamey, Niger

Aka Joseph (Santé Publique)

Département Etudes et Développement, CRESAC, Abidjan, Côte d'Ivoire

Mamadou Saidou (PharmD, PhD)

Département des Sciences Biologiques Appliquées, Faculté des Sciences de la Santé, Université Abdou Moumouni, Niamey, Niger

Doi:10.19044/esj.2019.v15n24p315 URL:http://dx.doi.org/10.19044/esj.2019.v15n24p315

Résumé

Introduction : Le choléra demeure un problème majeur de Santé Publique dans de nombreuses parties du monde et en particulier dans les pays d'Afrique subsaharienne. L'objectif de cette étude de faire une revue des données sur les épidémies de choléra, les facteurs de risques, microbiologiques et de lutte contre la maladie en Afrique sub-saharienne de 2010 à 2016. Materiels et méthode: Une revue de la littérature sur les épidémies de choléra 
en Afrique sub-saharienne de 2010 à 2016 a été conduite dans des banques de données ou bases de données et bibiothèques électroniques des pays ayant connu des épidémies. Les données annuelles de choléra dans les pays ayant connu des épidémies de 2010 à 2016 ont été rapportées. Résultats : Entre 2010 et 2016, 35 des 54 pays d'Afrique ont connu des épidémies de choléra. 1268 épisodes épidémiques ont été rapportés dont 13,04\% au Nigeria et 4,35\% au Burundi. Le nombre de cas notifiés était de 801022 dont 13232 décès (létalité globale $=1,65 \%$ ). Le Vibrio cholerae $\mathrm{O} 1$ et $\mathrm{O} 139$ sont les principaux agents étiologiques épidémiogènes. Les principaux facteurs de risques sont les pluies abondantes, les inondations, la contamination des sources d'eau et le manque d'assainissement. Conclusion : En dehors des souffrances éprouvées par les malades, les flambées de choléra provoquent la panique, désorganisent les structures économiques et sociales et freinent le développement des communautés touchées. Cependant, la mobilisation des secteurs de l'eau, de l'assainissement et de l'hygiène ainsi que le rensforcement des systemes de surveillance et riposte surtout au niveau transfrontalier sont des etapes essentielles pour la lutte contre les epidemies de cholera en Afrique subsaharienne.

Mots clés: Epidémies, Choléra, Facteur de risque, Afrique, Revue documentaire 


\section{Review of Reported Cholera Outbreaks in Sub-Saharan Africa, 2010 to 2016}

Alkassoum Salifou Ibrahim (MD, Msc, PhD candidat)

Département de Santé Publique, Faculté des Sciences de la Santé,

Université Abdou Moumouni, Niamey, Niger

Doutchi Mahamadou (MD, MPH Infectious Disease)

Faculté des Sciences de la Santé, Université de Zinder, Niger

Amadou Harouna (MD, Infectious Disease)

Département de Santé Publique, Faculté des Sciences de la Santé,

Université Dan Dicko Dan Koulodo, Maradi, Niger

Brah Souleymane (MD, MI)

Département de médecine et spécialités médicales, Faculté des Sciences de

la Santé, Université Abdou Moumouni, Niamey, Niger

\section{Djibo Issifou (MD, Msc, PhD candidat)}

Direction de la Surveillance et la Riposte aux Epidémies, MSP,

Niamey, Niger

Ibrahim Mahaman Lamine (Vet. PhD)

Lagaré A. (Biol, PhD candidat)

Centre de Recherche Médicale et Sanitaire de Niamey, Niger

Adehossi Eric (MD, MI)

Département de médecine et spécialités médicales, Faculté des Sciences de

la Santé, Université Abdou Moumouni, Niamey, Niger

Aka Joseph (Santé Publique)

Département Etudes et Développement, CRESAC, Abidjan, Côte d'Ivoire

Mamadou Saidou (PharmD, PhD)

Département des Sciences Biologiques Appliquées, Faculté des Sciences de

la Santé, Université Abdou Moumouni, Niamey, Niger

Abstract

Introduction: Cholera remains a major public health problem in many parts of the world and particularly in sub-Saharan African countries. The objective of this study is to review data on cholera epidemiology, risk, microbiological and disease control factors in sub-Saharan Africa from 2010 to 2016. Material and method: A literature review on cholera epidemics in sub-Saharan Africa from 2010 to 2016 was conducted using electronic 
databases from countries that have experienced epidemics. Annual cholera data for countries with outbreaks from 2010 to 2016 have been reported. Results: From 2010 through 2016, 35 of the 54 African countries have experienced cholera epidemics. An overall of 1268 outbreaks have been reported, of which $13.04 \%$ were recorded in Nigeria and $4.35 \%$ in Burundi. The number of cases reported was 801022 and 13232 deaths (overall CFR $=1.65 \%$ ). Vibrio cholerae $\mathrm{O} 1$ and $\mathrm{O} 139$ are the main etiological agents. The main risk factors are heavy rains, floods, contamination of water sources and lack of sanitation. Conclusion: In addition to the suffering of patients, cholera outbreaks cause panic, disrupt economic and social structures and hinder the development of affected communities. Mobilization of the water, sanitation and hygiene sectors is essential to ensure the benefits of patient care and cholera vaccination.

Keywords: Epidemics, Cholera, Risk factor, Africa, Review

\section{Introduction:}

Le choléra est une infection diarrhéique aiguë causée par la bactérie Vibrio cholerae $\mathrm{O} 1$ ou $\mathrm{O} 139$, après l'ingestion de nourriture ou d'eau contaminée.

En 2012, l'Organisation Mondiale de la Santé (OMS) estimait que chaque année, il y avait 3 à 5 millions de cas de choléra et plus de 100000 personnes mourraient de la maladie; la majorité $(99 \%)$ des cas et des décès survenant en Afrique subsaharienne et Asie du Sud (WHO, 2018) (Gaffa NH, Tauxe RV., Mintz ED, 2007).

De nos jours, le choléra demeure toujours un problème majeur de Santé Publique dans de nombreuses parties du monde; particulièrement dans les pays d'Afrique subsaharienne. En 2015, 42 pays avaient notifié un total de 172454 cas de choléra, dont 1304 décès, soit un taux de létalité (TL) global de $0,8 \%$. Sur l'ensemble de ces cas, $41 \%$ provenaient d'Afrique, $37 \%$ d'Asie et $21 \%$ de l'île d'Hispaniola (World Health Organization, 2016).

Le rôle du laboratoire de bactériologie est très important pour le diagnostic des cas isolés et des premiers cas d'un nouveau foyer épidémique. Le diagnostic de certitude repose sur l'identification de la souche de vibrion cholérique. La rapidité du diagnostic permettra de déclencher une action locale, nationale ou internationale immédiate ; une prise en charge prompte et adéquate étant déterminante pour prévenir la maladie ou limiter sa propagation.

Cependant, les raisons de la répétition, de la montée en intensité et de la propagation des épidémies demeurent mal comprises. Le contexte régional et surtout transfrontalier, marqué par des épidémies durant la même période a pu jouer un rôle. Le profil épidémiologique des épidémies dans les différentes régions d'Afrique sub-saharienne - dont certains districts sanitaires ont 
démontré des taux de létalité très élevés - évoque des difficultés structurelles dans la surveillance, la réponse précoce et dans la prise en charge initiale de ces épidémies (Mengel MA, 2014) (Alajo SO, Nakavuma J, Erume J, 2006). La compréhension de l'origine de ces variations géographiques à différentes échelles (régions sanitaires, districts sanitaires, zones transfrontalières) est une étape essentielle dans la lutte raisonnée et opérationnelle contre cette maladie. L'objectif de cette étude de faire une revue des données sur l'épidémiologie du choléra, les facteurs de risques, microbiologiques et de lutte contre la maladie en Afrique sub-saharienne de 2010 à 2016.

\section{Matériels et Méthode}

Une revue des épidémies de choléra en Afrique sub-saharienne dans des banques de données, bases de données et bibiothèques électroniques a été faite. Les données annuelles de choléra dans les pays ayant connu des épidémies de 2010 à 2016 ont été rapportées. Les sources de données comprennaient des rapports OMS publiés chaque année dans les bulletins épidémiologiques hebdomadaires (BEH), PubMed, le Réseau mondial des maladies infectieuses et épidémiologiques (GIDEON), le Programme de surveillance des maladies émergentes (ProMED), EMBASE et Google. les cumuls annuels de surveillance mondiale du choléra qui regroupent tous les cas de choléra et les décès signalés à l'OMS sur une base annuelle de 20102016 ont été pris en compte. Des recherches systématiques ont été effectuées à l'aide des termes de recherche "choléra", "diarrhée aqueuse aiguë », "épidémie de choléra" et "rapport d'investigation choléra". Les données ont été cumulées au niveau des pays, à l'exception du Niger, pour lequel les données au niveau infranational (régions et districts sanitaires) en raison de la disponibilité des rapports sur le choléra au niveau de la Direction de la Surveillance et la Riposte aux Epidémies (DSRE) du Ministère de la Santé ont été pris en compte.

Il a été passé en revue, les rapports d'investigation des épidémies de choléra, les procès-verbaux de réunions de crises portant sur les épidémies et, plus généralement, l'ensemble des documents d'intérêt sur le système d'alerte précoce et de la surveillance intégrée des maladies (Directives et recommandations nationales, plan de contingence, plan de riposte) disponibles sur les bases de données en ligne ou via OMS. Au total, 156 articles disponibles sur Pubmed, GIDEON, EMBASE et Google et 63 rapports ont été revus. Parmi ces articles, 93 ont traité des flambées épidémiques et 46 ont traité des facteurs de risques ou de récurrences des épidémies de choléra et 17 ont porté sur les investigations des épisodes épidémiques. Les éclosions d'épidémies ont été classées par facteurs de risque en fonction de la cause principale citée par l'auteur du rapport. 


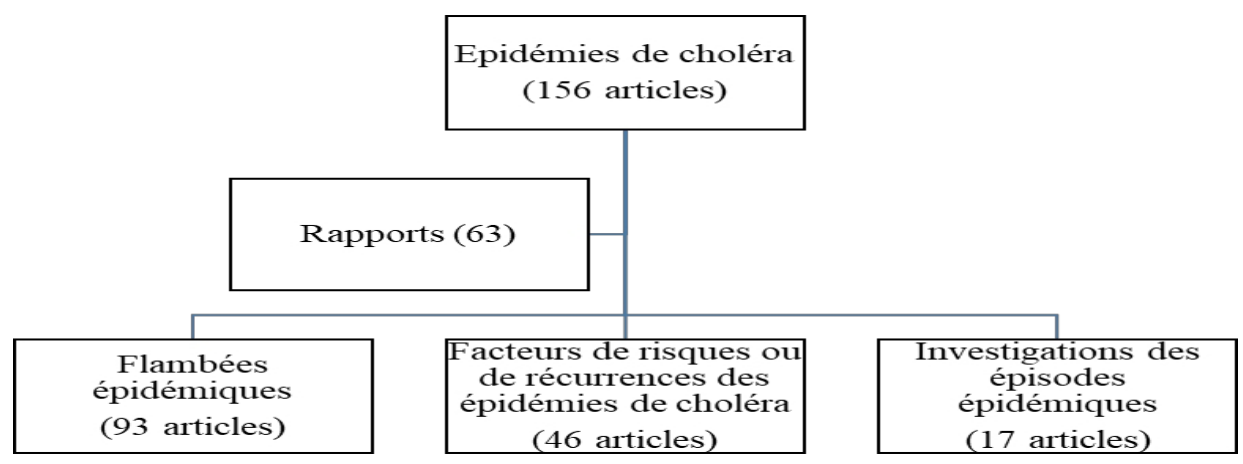

Figure 1 : Diagramme de flux

\section{Données épidémiologiques}

De 2010 à 2016, dans les 35 des 54 pays d'Afrique qui ont connu des épidémies de choléra, le nombre de cas suspects notifiés étaient de 801022 avec 13232 décès soit une létalité globale de 1,65\%. Sur un total de 1268 épisodes épidémiques rapportés, $13,04 \%$ se sont produits au Nigeria suivi du Burundi avec 4,35\% (Tableau I). Même si le nombre de cas a connu une diminution pendant cette période, l'incidence a dépassé 1000 cas par semaine dans certaines localités avec une propagation au-delà des frontières nationales.

Tableau I : Nombre de foyers d'épidémies et nombre de cas, décès et létalité rapportés en Afrique sub-saharienne, 2010-2016

\begin{tabular}{|c|c|c|c|c|}
\hline Pays & $\begin{array}{l}\text { Nombre de } \\
\text { foyers } \\
\text { d'épidémies } \\
\text { rapportées }(\%)\end{array}$ & $\begin{array}{l}\text { Nombre de cas } \\
\operatorname{suspects}(\%)\end{array}$ & $\begin{array}{l}\text { Nombre } \\
\text { de décès }\end{array}$ & Létalité(\%) \\
\hline Afrique du sud & $34(2,72)$ & $608(0,08)$ & 12 & 1,97 \\
\hline Angola & $41(3,26)$ & $88740(11,08)$ & 330 & 0,37 \\
\hline Benin & $48(3,80)$ & $4484(0,56)$ & 46 & 1,03 \\
\hline Burkina Faso & $14(1,09)$ & $169(0,02)$ & 9 & 5,33 \\
\hline Burundi & $55(4,35)$ & $3838(0,48)$ & 64 & 1,67 \\
\hline Cameroun & $48(3,81)$ & $37141(4,64)$ & 1631 & 4,39 \\
\hline RCA & $21(1,63)$ & $403(0,05)$ & 35 & 8,68 \\
\hline Congo & $28(2,17)$ & $3567(0,45)$ & 296 & 8,30 \\
\hline Cote d'ivoire & $41(3,26)$ & $2191(0,27)$ & 64 & 2,92 \\
\hline Djibouti & $14(1,09)$ & $2174(0,27)$ & 20 & 0,92 \\
\hline Ethiopie & $7(0,54)$ & $1682(0,21)$ & 21 & 1,25 \\
\hline Ghana & $41(3,26)$ & $50443(6,30)$ & 459 & 0,91 \\
\hline Guinée & $41(3,26)$ & $7673(0,96)$ & 164 & 2,14 \\
\hline Guinée Bissau & $28(2,17)$ & $4048(0,51)$ & 50 & 1,24 \\
\hline
\end{tabular}




\begin{tabular}{lllll}
\hline Kenya & $34(2,72)$ & $22454(2,80)$ & 221 & 0,98 \\
Liberia & $41(3,26)$ & $3202(0,40)$ & 2 & 0,06 \\
Malawi & $34(2,72)$ & $3947(0,49)$ & 80 & 2,03 \\
Mali & $28(2,17)$ & $2462(0,31)$ & 116 & 4,71 \\
Mauritanie & $14(1,09)$ & $46(0,01)$ & 3 & 6,52 \\
Mozambique & $48(3,80)$ & $21327(2,66)$ & 215 & 1,01 \\
Namibie & $21(1,63)$ & $488(0,06)$ & 13 & 2,66 \\
Niger & $48(3,81)$ & $9798(1,22)$ & 307 & 3,13 \\
Nigeria & $165(13,04)$ & $117425(14,66)$ & 3678 & 3,13 \\
RDC & $41(3,26)$ & $165667(20,68)$ & 1226 & 0,74 \\
Rwanda & $14(1,09)$ & $364(0,05)$ & 0 & 0,00 \\
Sénégal & $28(2,17)$ & $580(0,07)$ & 30 & 5,17 \\
Sierra Leone & $21(1,63)$ & $23501(2,93)$ & 331 & 1,41 \\
Somalie & $48(3,80)$ & $136561(17,05)$ & 2205 & 1,61 \\
Sud Soudan & $21(1,63)$ & $12534(1,56)$ & 296 & 2,36 \\
Tanzanie & $41(3,26)$ & $28890(3,61)$ & 264 & 0,91 \\
Tchad & $14(1,09)$ & $23662(2,95)$ & 633 & 2,68 \\
Togo & $48(3,80)$ & $617(0,08)$ & 22 & 3,57 \\
Uganda & $41(3,26)$ & $11701(1,46)$ & 293 & 2,50 \\
Zambie & $21(1,63)$ & $7322(0,91)$ & 71 & 0,97 \\
Zimbabwe & $34(2,72)$ & $1313(0,16)$ & 25 & 1,90 \\
Total & $1268(100,00)$ & $801022(100,0)$ & 13232 & 1,65 \\
\hline
\end{tabular}

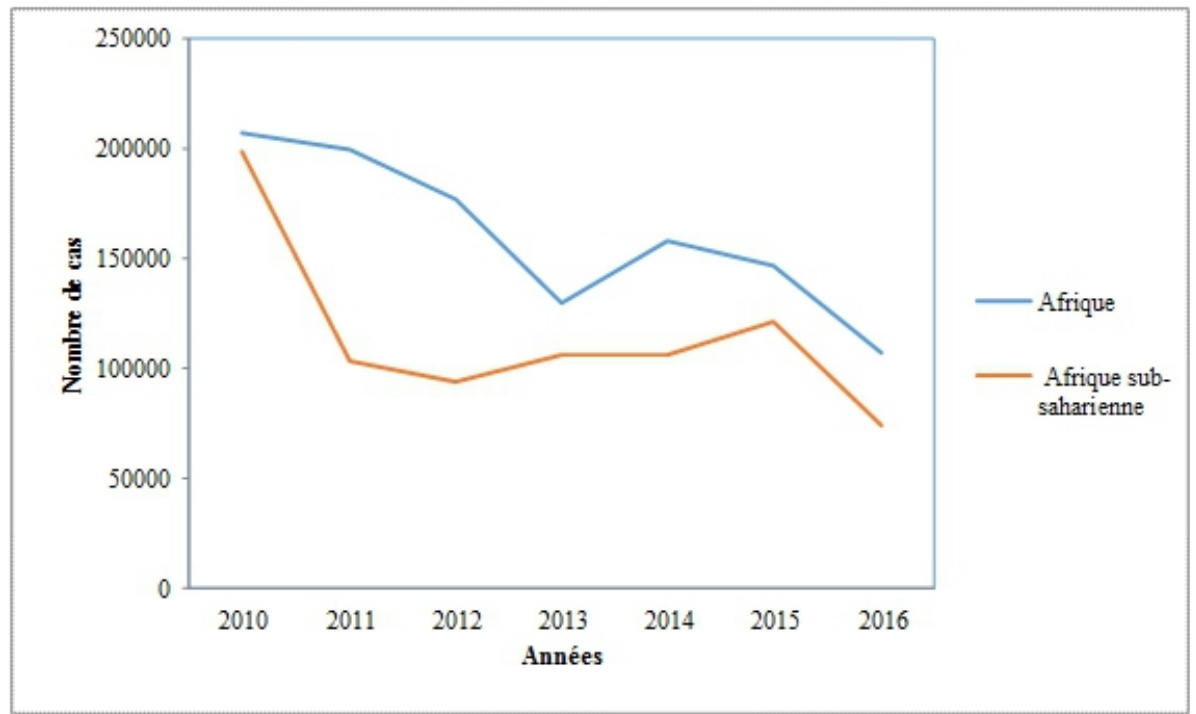

Figure 2 : Evolution des cas de choléra en Afrique et en Afrique sub-saharienne, 2010-2016 
L'évolution des cas de choléra de 2010 à 2016 a montré une variabilité dans la notification par année pour l'ensemble de l'Afrique et en Afrique subsaharienne. Pour les pays de l'Afrique subsaharienne, on note une diminution des cas de 2010 à 2012, suivi d'une légère augmentation en 2014 et 2015 et une diminution à partir de cette année (Figure 2).

La distribution par district Sanitaire des épidémies de choléra rapportées a fait ressortir une concentration de foyers multiples signalés se situant dans l'est de la République démocratique du Congo et l'Ouest de l'Ouganda, qui partagent une frontière commune. L'Afrique de l'Est et du SudEst ont également montré des concentrations élevées, ainsi que certaines parties de l'Afrique de l'Ouest. En Afrique du Sud, les foyers rapportés ont été limités aux provinces de l'Ouest, près de la frontière avec le Mozambique, qui ont signalé des foyers dans chaque province. La distribution sous-nationale des cas cumulatifs de choléra a montré un grand nombre de cas notifiés dans l'est de la République démocratique du Congo, au Mozambique et dans l'est de l'Afrique du Sud (Piarroux R, Marc M, 2002) (Justin L et al. 2018).

\section{Épidémiologie, facteurs de risque et morbidité du choléra en Afrique subsaharienne}

En 2016, 38 pays ont notifié 106587 cas avec 1762 décès dont $69 \%$ et $51 \%$ étaient respectivement notifiés en Afrique et Afrique sub-saharienne. Cependant, il existe une discordance entre ces chiffres et l'estimation de la charge de morbidité réelle au sein des pays vient du fait que de nombreux cas ne sont pas recensés en raison des insuffisances des systèmes de surveillance et des craintes de répercussions négatives sur le commerce et le tourisme (WHO, 2016). Il n'en reste pas moins que ces chiffres permettent de considérer le cholera comme un sujet de Santé Publique important qui nécessite une explication profonde des déterminants de son apparition et de sa persistance. Ainsi, de nombreuses études menées, notamment en Afrique subsaharienne ont montré l'implication des plusieurs facteurs de risques expliquant l'éclosion, la diffusion et la persistance des épidémies de choléra (Ali M, Nelson AR, Lopez AL, Sack DA, 2015) (Isaäcson D. L, Swerdlow M, 2005).

Le mode de transmission de la maladie est étroitement lié à un accès inapproprié à l'eau potable et à des installations d'assainissement. La maladie est fréquente dans les zones à risque typiques des bidonvilles périurbains, qui ne disposent d'aucune infrastructure de base, ou les camps de réfugiés ou de personnes déplacées, où les besoins minimums en eau potable et en assainissement ne sont pas assurés. Les crises humanitaires, qui ont notamment pour conséquence l'interruption des systèmes d'approvisionnement en eau et d'assainissement et les déplacements de populations dans des camps mal équipés et surpeuplés, peuvent augmenter le 
risque de transmission du choléra ou amplifier une épidémie existante, si jamais le bacille est présent ou s'il est introduit. Cependant, il n'y a jamais eu d'épidémie à partir de cadavres de personnes non infectées (Ndour C T et al., 2006) (Kelias P M et al, 2014) (Sounkalo Dao et al.,2009)

Tableau II : Catégorisation des facteurs de risque de choléra en Afrique subsaharienne, 2010-2016

\section{Facteurs de risque \\ 1. Pluies et inondations \\ 2. Contamination d'une source d'eau}

3. Mauvaise hygiène

4. Absence potable

5. Présence de camps de refugiés

6. Aliments

7. Transport

8. Zone de conflit

9. Urbanisation/ densité de population

10. Saisonnalité

11. Rites funéraires/ regroupements (fêtes, marchés...)

12. Prisons

\section{Causes}

Les pluies abondantes et les inondations perturbent les systèmes d'approvisionnement en eau et contribuent à la propagation du choléra.

La contamination par vibrion cholerae d'une source d'eau comme les lacs, les rivières ou les canalisations d'eau domestique

Le manque d'assainissement de base telle que la disponibilité des latrines, le lavage des mains.

La disponibilité limitée de l'eau potable oblige les populations à utiliser de l'eau contaminée à des fins domestiques.

Les camps de réfugiés ou de personnes déplacées sont surpeuplés et disposent de ressources limitées en eau potable et latrines.

Les mollusques et crustacés contaminés ou légumes crus non lavés

La propagation ou l'amplification du choléra par les voyageurs au-delà des frontières nationales

Les zones de guerre peuvent augmenter le risque d'épidémies parce que les infrastructures sont endommagées et que les gens n'ont pas accès aux formations sanitaires ou à des soins médicaux appropriés

Dans les villes et les bidonvilles, les personnes vivant à la proximité immédiate augmentent le fardeau de l'assainissement et facilitent la transmission.

Le risque de survenue du choléra est augmenté pendant certaines saisons de l'année (saison de pluie)

Certains rites funéraires traditionnels comprennent le lavage du défunt et la préparation d'un grand repas. Cette situation, combinée au fait que les personnes endeuillées voyagent souvent, permet la propagation de la maladie.

Les détenus n'ont pas toujours le contrôle de leurs besoins en matière d'assainissement et vivent dans un contexte de promiscuité

Les douze (12) facteurs de risque les plus fréquemment cités dans les rapports sont énumérés avec des descriptions résumées de la façon dont ils ont contribué aux éclosions des épidémies (Tableau II) (World Health Organization. Cholera, 2014) (Mengel MA, Delrieu I, Heyerdahl L, Gessner 
BD, 2014) (WHO, 2016) (Ali M, 2015) (Justin L, 2018) (Universalis, 2018).) (Felix H, 1971) (Carme B, et al., 1983) (Gérard R, Dejours H, 1983).

Tableau III : Répartition des facteurs de risque identifiés par région de l'Afrique subsaharienne, 2010-2016

\begin{tabular}{|c|c|c|c|c|}
\hline Facteurs de risque & $\begin{array}{l}\text { Afrique } \\
\text { Centrale } \\
\end{array}$ & $\begin{array}{l}\text { Afrique } \\
\text { de l'Est }\end{array}$ & $\begin{array}{l}\text { Afrique } \\
\text { du Sud }\end{array}$ & $\begin{array}{l}\text { Afrique de } \\
\text { l'Ouest }\end{array}$ \\
\hline Pluies et inondations & $41(40)$ & $104(54)$ & $83(55)$ & $101(55)$ \\
\hline $\begin{array}{l}\text { Contamination d'une source } \\
\text { d'eau }\end{array}$ & $12(12)$ & $9(5)$ & $26(17)$ & $29(16)$ \\
\hline Mauvaise hygiène & - & $21(11)$ & $20(13)$ & $27(15)$ \\
\hline Absence d'eau potable & $18(18)$ & $9(5)$ & $8(5)$ & $5(2)$ \\
\hline Présence de camps de refugiés & $3(3)$ & $5(2)$ & $3(2)$ & $3(2)$ \\
\hline Aliments & $5(4)$ & $32(16)$ & - & $2(1)$ \\
\hline Transport & $14(13)$ & - & $2(1)$ & $6(3)$ \\
\hline Zone de conflit & - & $9(5)$ & $5(3)$ & $6(3)$ \\
\hline $\begin{array}{l}\text { Urbanisation/ densité de la } \\
\text { population }\end{array}$ & - & - & - & $3(2)$ \\
\hline Saisonnalité & $3(3)$ & - & $2(1)$ & - \\
\hline $\begin{array}{l}\text { Rites funéraires/ regroupements } \\
\text { (fêtes, marchés...) }\end{array}$ & $2(1)$ & $2(1)$ & $2(1)$ & $2(1)$ \\
\hline Prisons & $5(4)$ & $2(1)$ & $2(1)$ & - \\
\hline Total & $101(100)$ & 191(10) & 149(10) & $182(100)$ \\
\hline
\end{tabular}

Les différentes régions ont montré la variabilité des facteurs de risque identifiés pour les épidémies de choléra (Tableau III). En Afrique de l'Ouest, Afrique du Sud et de l'Est, le facteur de risque le plus souvent cité était les fortes pluies et les inondations, qui représentaient respectivement 55\%, 55\% et $54 \%$ de tous les rapports sur les facteurs de risque. En revanche, elles n'ont représenté que $40 \%$ des rapports en Afrique central. Les rapports identifiaient la contamination d'une source d'eau dans $17 \%$ et $16 \%$ respectivement en Afrique du Sud et de l'Ouest. La présence de camps de réfugié ou de personnes déplacées à l'intérieur du pays était représentée dans 3\% en Afrique centrale et $2 \%$ en Afrique de l'Ouest, du sud et de l'Est respectivement. En Afrique centrale, aucun foyer n'a été attribué à la mauvaise hygiène. En Afrique de l'Ouest, du Sud et du centre, la contamination des sources d'eau était le deuxième facteur de risque le plus courant, représentant respectivement $16 \%$, $17 \%$ et $12 \%$ du total des rapports. En Afrique centrale, l'absence d'eau potable était le deuxième facteur de risque, représentant $18 \%$ des facteurs de risque signalés (David C et al., 2006) (Bwire G et al., 2016). 


\section{Principales caractéristiques microbiologiques du vibrio cholerae et son environnement naturel en Afrique subsaharienne}

Des études en Afrique, sur des expérimentations en microcosme simulant des environnements marins, estuariens, d'eaux saumâtres ou d'eaux douces, ont élargi les connaissances sur la physiologie de Vibrio cholerae. Cela a permis entre autres de quantifier la relation entre l'abondance de la bactérie et la température de l'eau : il est, en effet, plus fréquent d'isoler la bactérie durant la période chaude. D'autres travaux ont montré que la fréquence d'isolement de la bactérie est également reliée avec la salinité du milieu, avec des valeurs optimales pour des salinités comprises entre 0,2 et 2\%o (Colwell RR, Huq A., 1994). Une autre étude au Tchad a montré que sur 1011 échantillons d'eau prélevés sur quatre types de sources d'eau (rivières, puits, égouts et lac), 244 étaient positifs Vibrio cholerae non-O1 et non-O139, tandis qu'aucun n'était positif au Vibrio cholerae O1. Aussi, l'analyse de régression binomiale a montré un risque accru d'infection par Vibrio en avril, mai et juillet et que par rapport à la détection de Vibrio, une augmentation significative de la détection a été observée dans les puits et les drains d'égouts (Colwell RR, Rita R, 2004).

\section{Impact des épidémies de choléra et moyens de lutte en Afrique sub- saharienne}

En dehors des souffrances éprouvées par les malades, les flambées de choléra provoquent la panique, désorganisent les structures économiques et sociales et peuvent freiner le développement des communautés touchées. De même les catastrophes naturelles ou provoquées par l'homme, ainsi que les conditions régnant dans les camps de réfugiés surpeuplés, peuvent augmenter beaucoup les risques d'épidémies. Il en résulte souvent des flambées explosives avec des taux de létalité élevés. A la suite de la crise au Rwanda en 1994 par exemple, il y a eu 73605 cas de choléra, dont 1612 décès au cours des flambées qui se sont produites dans les camps de réfugiés de Goma, au Congo (Didier B, Patrick G, Martine P, Guy M. et al, 2009). Bien qu'elles ne soient pas toujours aussi mortelles, les flambées posent un grave problème de Santé Publique en Afrique et entraînent des perturbations considérables de la vie sociale et économique, ainsi que des pertes en vies humaines.

Cependant, les restrictions de routine sur les mouvements de personnes, y compris les mesures de quarantaine ou "cordon sanitaire", se sont révélées inefficaces dans la lutte contre le choléra et sont donc considérées comme inutiles. L'OMS ne recommande pas le dépistage systématique, la mise en quarantaine ou d'autres restrictions similaires pour les voyageurs en provenance de régions touchées par une épidémie de choléra. Les autorités doivent fournir des soins médicaux appropriés aux patients atteints du choléra, le cas échéant, et sont encouragées à fournir aux voyageurs des informations 
sur les risques d'infection par le choléra, la prévention des risques (OMS, 2018). En dehors de stratégies classiques de lutte contre le choléra, la vaccination est utilisée en appui dans des zones à haut risque de cholera. Cependant, la mobilisation des secteurs de l'eau, de l'assainissement et de l'hygiène est essentielle pour garantir les avantages à court terme des vaccins anticholériques. Une étude menée au Malawi a révélé que les zones à risque épidémiologique élevé de choléra ayant fait l'objet d'une vaccination n'ont pas été touchées par des épidémies malgré une épidémie nationale en 2017 2018 (Maurice MB, Lorenzo P, Bruce R, Storn Ka et al,2018). Une autre étude de modélisation sur l'impact des interventions ciblé de la réponse aux épidémies de choléra a montré que l'utilisation du Vaccin Cholérique Oral (VCO), les antibiotiques et les interventions de traitement de l'eau dans un rayon approprié autour des cas pourraient être un moyen efficace et efficient de combattre les épidémies de choléra. Ils peuvent fournir une approche complémentaire et efficace aux campagnes d'intervention de masse et peuvent s'avérer particulièrement utiles pendant la phase initiale d'une épidémie, lorsqu'il y a peu de cas et peu de ressources disponibles, ou afin de raccourcir les tendances souvent prolongées des épidémies de choléra (Flavio F, Enrico B, Francisco J L, Nathan N, 2018).

\section{Conclusion}

Le choléra demeure toujours un problème majeur de Santé Publique dans les pays en développement. La létalité générale reste élevée chez les groupes vulnérables dans les zones à haut risque. Plusieurs sérogroupes ont été définis, dont seulement deux (O1 et O139) sont susceptibles de provoquer le choléra. En dehors de stratégies classiques de lutte contre le choléra, la vaccination est utilisée en appui dans des zones à haut risque de choléra. Cependant, la mobilisation des secteurs de l'eau, de l'assainissement et de l'hygiène ainsi que le renforcement des systèmes de surveillance et riposte sont des étapes essentielles pour la lutte contre les épidemies de choléra en Afrique subsaharienne.

\section{References:}

1. WHO Media Centre (2018). Preventing and trating cholera: Fact file on cholera. s.l.: Geneva: world health organization,

2. Gaffa NH, Tauxe RV., Mintz ED. (2007). Cholera: a new homeland in Africa? Am J Trop Med Hyg ; 77 : 705-713.

3. World Health Organization (2016). Cholera, 2014.Wkly Epidemiol Rec ; 91(39): 433-440.

4. Mengel MA. (2014). Cholera in Africa: new momentum in fighting an old problem. Trans R Soc Trop Med Hyg [Internet]. 108(7):391-2. 
Available from: http://www.ncbi.nlm.nih.gov/pubmed/24836060 https://doi.org/10.1093/trstmh/tru077 PMID: 24836060

5. Alajo SO, Nakavuma J, Erume J. (2006). Cholera in endemic districts in Uganda during El Niño rains: 2002-2003. Afr Health Sci.; 6:93 7 . https://doi.org/10.5555/afhs.2006.6.2.93 PMID: 16916299

6. Piarroux R, Marc M. (2002). Le choléra : épidémiologie et transmission. Expérience tirée de plusieurs interventions humanitaires réalisées en Afrique, dans l'Océan Indien et en Amérique Centrale. Bull Soc Pathol Exot; 95(5):345-350.

7. Justin L, Sean MM, Francisco J L, Heather S M et al. (2018). Mapping the burden of cholera in sub-Saharan Africa and implications for control: an analysis of data across geographical scales. The Lancet; http://dx.doi.org/10.1016/ S0140-6736(17)33050-7.

8. WHO. (2016). Relevé épidémiologique hebdomadaire, 16 décembre 2016, vol. 91, 51/52. pp. 601-624. [En ligne]. Disponible sur http://www.who.int/wer/2016/wer9151_52/fr/. [Citation : 2105 2018.]

9. Ali M, Nelson AR, Lopez AL, Sack DA. (2015). Updated Global Burden of Cholera in Endemic Countries. PLoS Negl Trop Dis; 9(6): e0003832. doi:10.1371/journal.pntd.0003832.

10. Isaäcson D. L, Swerdlow M. (2005). The Epidemiology of Cholera in Africa in Vibrio cholerae and Cholera: Molecular to Global Perspectives, Washigton, DC; 297-307.

11. Ndour C T, Manga N.M, Ka R, Dia/Badiane N.M. et al. (2006). L'épidémie de choléra de 2004 à Dakar: aspects épidémiologiques, cliniques et thérapeutiques. Med Trop; 66:33-38

12. Kelias P M, Mathew K, Maurice M, Sikhona C. et al. (2014). Cholera outbreaks in Malawi in 1998-2012: social and cultural challenges in prevention and control. J Infect Dev Ctries;8(6):720-726. doi:10.3855/jidc.3506.

13. Sounkalo Dao et al. (2009). « Les épidémies de choléra au Mali de 1995 à 2004 ». Santé Publique; 3(21):263-269. DOI 10.3917/Spub.093.0263.

14. World Health Organization. Cholera (2014). Wkly Epidemiol Rec [Internet]. 2015; 90(40):517 28 . Available from: http://www.ncbi.nlm.nih.gov/pubmed/26433979 PMID: 26433979

15. Mengel MA, Delrieu I, Heyerdahl L, Gessner BD (2014). Cholera outbreaks in Africa. Current Topics in Microbiology and Immunology. 2014. p. 117-44.

16. Universalis. (2018). Cholera. [En ligne] disponible sur: https://www.universalis.fr/encyclopedie/premiere-pandemie-decholera/\#i_11349. Accédé le 13/09/2018 
17. Felix H. (1971). Epidémie de choléra en Afrique. Note d'information sur l'évolution de la situation entre Août et Décembre 1970. Prese Médicale, Vol. 79;475-8

18. Carme B, Mavumu M N, Tripe J.-F, Yala F et al. (1983). L'implantation et l'extension du choléra en Afrique Noire : 1970 1986. Rev. Med. Congo, 2(34):17-29

19. Gérard R, Dejours H. (1983). L'Africanisation du choléra. In: Cahiers d'outre-mer. $\mathrm{N}^{\circ}$ 162. 41e année, Avril-juin; 105-138.

20. David C. Griffith Louise A. Kelly-Hope, and Mark A. Miller. (2006). Am J. Trop. Med. Hyg. 2006, Vol. 75(5), pp. 973-977.

21. Bwire G, Mwesawina M, Baluku Y, Kanyanda SSE, Orach CG. (2016). Cross-Border Cholera Outbreaks in Sub-Saharan Africa, the Mystery behind the Silent Illness: What Needs to Be Done?. PLoS ONE; 11(6): e0156674. doi:10.1371/journal.pone.0156674.

22. Colwell RR, Huq A. (1994). Environmental reservoir of Vibrio cholerae. The causative agent of cholera. Ann N Y Acad Sci; 740:4454.

23. Colwell RR, Rita R. (2004). Infectious disease and environment: cholera as a paradigm for waterborne disease. International Microbiology; 15(2)7:285-289.

24. Didier B, Patrick G, Martine P, Guy M. et al. (2009). Cholera Epidemics, War and Disasters around Goma and Lake Kivu: An EightYear Survey. Plos neglected tropical disease, www.plosntds.org,3(5): e436.

25. OMS. (2018). Politique et recommandations de l'OMS concernant les flambées de choléra: Prévention et lutte. [Disponible] sur http://www.who.int/topics/cholera/control/fr/, [accédé le 05/09/2018]

26. Maurice MB, Lorenzo P, Bruce R, Storn Ka et al. (2018). Oral cholera vaccine in cholera prevention and control. Malawi Bull World Health Organ; 96:428-435 doi: http://dx.doi.org/10.2471/BLT.17.207175.

27. Flavio F, Enrico B, Francisco J L, Nathan N. (2018). The potential impact of case-area targeted interventions in response to cholera outbreaks: A modeling study.PLoS Med; 15(2):e1002509. https://doi.org/ 10.1371/journal.pmed.1002509. 